\title{
Paesaggi intangibili, voi dite...
}

Franco Zagari

Architetto, paesaggista

info.zagari@gmail.com

\begin{abstract}
... E dire che è proprio in remoto che avremmo scoperto il fascino dell'assenza ...

Paesaggi intangibili, voi dite... Con questo termine 'intangibile' definiamo alcuni paesaggi materiali e immateriali che sono espressioni di diritti acquisiti nella nostra storia al prezzo di lotte e sacrifici, di cui si vuole preservare il valore in quanto caratterizzanti della nostra identità. Come sapete ho sempre sostenuto che la sostanza del paesaggio, se inteso come progetto, abbia forme e contenuti che è non solo possibile, ma doveroso cercare di definire, perché è anche una questione eminentemente politica, da proporre al Paese come un impegno prioritario quotidiano.
\end{abstract}

\section{Parole chiave}

Bellezza, dignità del lavoro, capacità di ascolto, principi di orientamento e qualità di nuova centralità

\begin{abstract}
... And to think that it is precisely by working remotely that we would have discovered the fascination of absence...

Intangible landscapes, you say... With this term 'intangible' we define some material and immaterial landscapes that are expressions of rights acquired in our history at the price of struggles and sacrifices, whose value we want to preserve because they characterize our identity. As you know, I have always sustained that the substance of the landscape, if understood as a project, has forms and contents that it is not only possible, but dutyful to define, because it is also an eminently political issue, to be proposed to our country as a daily priority commitment.
\end{abstract}

\section{Keywords}

Beauty, dignity of work, listening skills, principles of orientation and quality of new centrality 
Questo vale per definire documenti di principi generali, come opere, fra cui un quadro che desidero ricordare in particolare, per rendergli dovuto omaggio, il Quarto Stato di Giuseppe Pellizza da Volpedo (dipinto fra il 1891 e il 1901), che è una pietra miliare del simbolismo e del socialismo in Italia. Si noti che qui il paesaggio è costituito da una massa umana in marcia, sono solo persone, uomini, donne e bambini, il proletariato, sono loro che danno forma allo spazio attorno, senza descriverlo. Fortemente unificante è il gioco delle luci e delle ombre, una gamma cromatica calda, illuminata dal sole, contenuta fra le tonalità del cremisi e del rosso. Questa patina dà un tono rassicurante al messaggio politico, che è decisamente positivo.

Forte di questa immagine che suggerisce speranza e solidarietà inizio questa mia riflessione provando a dirvi cosa vuol dire per me la parola 'intangibile': irriducibile, incomprimibile, non mediabile e appartenente a diverse altre famiglie concettuali che vertono sulla contaminazione, come categoria stimolante di. pensiero, che se riferita al Landscape apre bene una discussione da uno dei crocevia più creativi della trasformazione dello spazio degli ultimi cinquanta anni.

È una parola curiosa, piuttosto desueta nel suo uso, che esprime quella particolare necessità che si forma e si sviluppa nell' ambiente di lavoro di un progetto di paesaggio, di 'mettere in tensione' una scena di azioni umane fisiche e mentali, un ambito coerente ancorché dialettico, di elementi e di azioni utili a comprendere, modificare e valorizzare le sue vocazioni. Complicato? Fabio Bianconi e Marco Filippucci per aiutarci se la sono cavata con poco più di 1.200 pagine con il loro Digital Draw Connections Representing Complexity and Contraddiction in Landscape, uno scoop editoriale formidabile (Springer, Cham, Switzerland, 2021).

Fra i significati di 'irrinunciabile' e di 'ambiguità' nella grande e tempestosa casa del progetto di paesaggio l'esplorazione è vasta, ma non imprecisa. Sullo sfondo a mio avviso molto si deve a Robert Venturi, il grande maestro di una postmodernità che fece del pop e della landart un nuovo cantico il cui slogan avrebbe potuto essere "un monumento alla portata di tutti". Dunque il lettore è avvertito: ora è a lui la parola.

E non è certo un caso che il suo itinerario di visita sia labirintico e inizi da una "selva oscura". Vi prego di seguirmi in un incipit che parte da una interpretazione leggendaria di quanto certamente fu, II nostro spazio pubblico più antico.

\section{Valori: Bellezza, Lavoro, Ascolto}

Nella storia dell'umanità alcuni valori attraversano i secoli con nomi diversi, ma simili nella sostanza. 
....Fra tante isole e penisole -dissi- lungo questa costa frastagliata costellata di resti di naufragi, ero sicuro che dovessi esserci anche tu, in attesa, Regina indiscussa del male universale (Regina? II diavolo in 'quote rosa'? E perché no? Che sia una autosuggestione, una precoce intuizione di un mutamento in atto nel serpente stesso? La condizione di innocenza del paradiso essendo un concetto troppo sofisticato perché il consesso umano potesse comprenderlo e accoglierlo rapidamente...."

Possiamo quindi dire senza timore che il Paradiso terrestre è il nostro spazio pubblico ancestrale più antico. Immaginatelo per lo più come un gigantesco albero dai fiori vermigli, fluttuante nel vuoto come una Tillandsia, ma senza peso, che ci guida senza speranza a ritornare eternamente indietro, a riflettere sull'origine del nostro complesso di colpa, del quale spazi con continue biforcazioni sperimentano agnizioni, antidoti, anatemi. Ecco il primo spazio 'intangibile' della storia umana, nasce da un diritto acquisito da soggetti selezionati per censo, classe sociale, appartenenza a una determinata confessione religiosa, economica, politica. E chi altri potevano essere come primi, se non loro, i due prototipi per antonomasia, Adamo e Eva, i figli eletti, chiamati a godere di un privilegio assoluto, ma dalle severe regole molto definite e certamente non revocabili.
Che voi a questo punto possiate avere una leggera sensazione di vertigine è nella norma di questo tipo di incontri, non preoccupatevene. Spesso l'immagine più forte di uno spazio urbano è l'incontro fra le persone che lo frequentano, nei comportamenti più semplici come in quelli più complessi.

Una considerazione però dovrebbe essere fatta, che può qui essere soltanto enunciata per ragioni di spazio, riguarda l'idea di tangibilità se riferita a una particolare fase progettuale, sia essa di salvaguardia, di gestione o di innovazione. Direi di soprassedere all'argomento degli effetti dovuti alla creatività dell'autore, se singolare o plurale, maschile o femminile, credo che vi sia già una solida bibliografia al riguardo: credo che un paesaggio possa essere o diventare intangibile in uguale misura nei due casi. Ma credo soprattutto che oggi il problema non sia qui, che dovrebbe spostarsi sulla questione generale della modificabilità o meno di un contesto.

La intangibilità dovrebbe riguardare le scelte di quel mandato che una collettività abbia ritenuto di adottare in seguito a ponderati processi partecipativi, decisioni che se prese in sé, senza una adeguata motivazione del progetto, rischiano di seguire una deriva molto schematica e addirittura di poter ottenere il contrario di quanto desiderato. Due pesanti opzioni sono infatti profondamente radicate nell'opinione pubblica:

- in primo luogo per quanto riguarda il patrimonio, la cui natura eminentemente conservatrice per dogma dovrebbe spettare a opere su contesti storici, senza temere di adottare il falso, purché i requisiti stilistici e la visibilità siano affini alle tradizioni del contesto;

- in secondo luogo la sostenibilità ambientale, che debba sempre essere ridotta a fattori quantitativi di superfici basati su zoning, aree, standard, verde mq/abitante, parcheggi mq/abitante. 


\section{Valori: Religio. La Passione. Deserto del Neghev (Israele), Cinecittà}

Ora, al mio quadro, per procedere con equilibrio, manca una ouverture e una grande promenade.

Esattamente cinquanta anni fa escono due film che hanno fatto epoca, nei quali ho trovato una vena che ha stabilito per me un nuovo modo di guardare il paesaggio. Sono Jesus Christ Superstar di Norman Jewison, in particolare l'Ouverture, e Fellini 8 e 1/2, in particolare il grande Carosello finale. Sono in fondo due musical, che culminano in due episodi estremi, all'inizio l'imminenza del sacrificio del Cristo nella vestizione di tutti gli interpreti, che da hippy multicolori diventano rapidamente sacerdoti, soldati, plebe, da cui l'improvvisa accettazione della caduta di qualsiasi filtro intellettuale. Nella Roma che segue, ciò che resta de La dolce vita, il crollo simbolico dell'astronave, sempre più imperscrutabile e ostile nei suoi reconditi significati e per contro la restituzione di tutti gli interpreti a quello che sono nella vita: sentimenti di privacy ordinaria.

Ecco due storie raccontate con grande originalità, dove il tempo si annulla - vi viene da chiedere se sapete se e quando questi due film saranno girati, visti, distribuiti -. entrambi sono nati come un inno alla libertà, ma i miei allievi ne ignoravano l'esistenza. Ecco che un prezioso spazio della memoria come conquista si va rarefacendo...

\section{Valori: Dichiarazioni dei diritti dell'uomo}

Alla fine del Settecento un vasto movimento rivoluzionario genera grandi cambiamenti pro e contro in tutto il mondo occidentale, per quanto riguarda una forte promiscuità di idee rivoluzionarie e riformiste, che maturano in almeno un decennio di alacre trasformismo. II punto più alto di arrivo del confronto fra le rispettive posizioni è a cominciare dalle dichiarazioni dei diritti dell'uomo che sono alla base dei pronunciamenti di indipendenza delle repubbliche americana e francese. Su questo dobbiamo interrogarci ancora oggi, chiederci se e come fu che l'Europa mettesse il piede in fallo, con l'inizio di un'eclisse che non si spegnerà più. La rivolta contro la tirannia del regno, del clero e della nobiltà deflagrerà in una delle prime grandi battaglie urbane nel Iuglio 1830 e troverà in marcia alla testa del popolo i ceti della nuova borghesia, saranno gli eroi di Delacroix e Hugo.

Fin dall'inizio le cose non sono state così facili. Covava negli Stati Uniti d'America la questione della schiavitù che nel secolo seguente ha portato a una disastrosa guerra civile. E in Francia i contadini non fecero parte dei rappresentanti del popolo eletto nella prima Assemblea.

\section{La libertà che guida il popolo}

La libertà che guida il popolo (1830), di Eugène Delacroix, e il romanzo Les misérables (1852) di Victor Hugo rimangono le opere più rappresentative di un tempo che sarà comunque l'anima della Francia.

Questa storia torna più volte in remake con varie forme e diversi dispositivi, fino a una geniale sequenza di film, in particolare Les misérables, film del 2012 diretto dall'allora giovanissimo Tom Hooper, monumentale capolavoro basato sull'omonimo musical tratto dal romanzo di Hugo.

Les misérables Film omonimo ambientato nella banlieue parigina diretto da Ladj Ly, che ha avuto un ennesimo successo poco dopo, nel 2012 Non credo sia accettabile di non ritenere questi paesaggi fra quanti abbiamo il dovere di custodire perché siano veramente intangibili, perché siano pienamente prototipi e campioni di democrazia, perché sono la culla della Dichiarazione Universale dei Diritti dell'uomo, un documento sui diritti della persona, che è stato adottato dall'Assemblea generale delle Nazioni Unite nella sua terza sessione, il 10 dicembre 1948 a Parigi con la risoluzione 219077A. Così recita I'Articolo 1:

\footnotetext{
"Tutti gli esseri umani nascono liberi ed eguali in dignità e diritti. Essi sono dotati di ragione e di coscienza e devono agire gli uni verso gli altri in spirito di fratellanza".
} 

che sia in equilibrio fra tradizioni del luogo e visioni del futuro di una sua possibile trasformazione. Proprio come virus potremmo rischiare di chiamarli, per stare in metafora con un piede nella nostra recente attualità, lo dico perché l'intangibilità è quella condizione che abbiamo visto tante volte in immagini affascinati ad esempio dalla difesa di una cellula da un'aggressione biologica. Questo per lo meno dando al paesaggio lo spazio di un pregiudizio, che in principio potrebbe essere positivo sulle attività, i flussi e i comportamenti che lo definiscono.

Non soluzioni ma dubbi ci vengono allora prontamente incontro. È più corretto dire che avremmo bisogno di tutto ciò se avessimo le spalle così robuste per poterlo sopportare.

\section{Valori: Libertà}

"Viva la Libertà" è l'unico valore che anche da solo potrebbe rappresentare caso per caso quel sentimento ideale cui concorrono individui e comunità, quando esprimano una dichiarazione di volontà, con un sentimento che è il più diretto, l'amore per la propria indipendenza.

Questo è un patto evidentemente non contrattabile oltre ai termini di un suo statuto, che naturalmente varia nel tempo, ma che può essere confermato in ogni momento con precisione esprimendolo con una volontà esplicita.

Questo principio, apparentemente così semplice, è alla base di importanti continue variazioni dell'assetto fisico del territorio e riguarda l'evoluzione di quelle qualità di orientamento e di centralità che definiamo come obiettivi caratteristici dei paesaggi.

Fra i casi che io porgo alla vostra attenzione alcuni sono opere d'arte che sono state dichiarate intangibili, altri sono immateriali, come lo è la Dichiarazione dei diritti dell'uomo, perché principi fondamentali dell'umanità. II concetto di intangibilità garantisce le azioni di salvaguardia, di gestione, di valorizzazione quando opera per difendere e affermare principi fondamentali della natura umana. L'intangibilità è un concetto che nasce qui, con due volti:

1. il primo sembrerebbe contraddire il significato dell'etimo, ci rinvia a un guscio resistente, che protegge valori interni come uno scrigno, per questo non è negoziabile, denota le proprietà di un campo privilegiato di doveri e di diritti, cui si è ammessi di regola per merito, o se è necessario per epiche lotte, e l'estensione di questa proprietà può spingersi anche molto lontano, difesa da una filigrana di norme e commi che gli conferiscono il carattere e la struttura di un'istituzione riconosciuta. Questo succede quando un perimetro stabilisce in modo inequivocabile le specifiche e gli obblighi di determinati comportamenti; è il momento nel quale l'appartenenza a un luogo comincia a contare in funzione di un patto che ne rappresenta i valori essenziali e, di conseguenza, che le famiglie entrano nella grande competizione per il controllo politico di un territorio intervenendo in principio su matrimoni, successioni e eredità e procedendo poi con un approccio che è sempre più difficile distinguere dalla ragione di stato.

2. il secondo volto dell'intangibilità riguarda soprattutto la storia dei movimenti libertari, che potremmo chiamare dei diritti acquisiti di cittadi- 
nanza, che sono stabiliti con la stessa funzione dei primi, ma questa volta dall'interno di un clan familiare.

\section{Una storia dal doppio spettro}

Cerchiamo ora di passare a una storia che ha un doppio spettro, dall'esterno verso l'interno e viceversa. La piazza che scelgo è lo scenario di un grande spettacolo, il progetto della Piazza del Sol a Madrid, la piazza più nota e popolare di Spagna, è un prototipo di uno spazio pubblico di nuova generazione, molto progettato sul tempo, la scansione del movimento del pubblico che qui alterna una frenetica apertura e chiusura di entrate e uscite, di sopra e sotto, di dentro e fuori, di spazi di stazione, di serra, di mercato, tutto con un ritmo iper-veloce. Un vuoto anch'esso pervaso da una umanità contagiata da una fretta che solo le tre linee di metro che qui si danno sotterraneo congresso può motivare, che è movimento anch'esso, un'eco di quanto accade in superficie ma su distanze che coprono l'intera città storica. Un glutine di vegetazione fra dentro e fuori, costantemente curato dall'Orto botanico di Madrid articola questi spazi con una musicalità che in questo momento, se richiesto, io affiderei al ricordo di Luciano Berio.

Fuori terra un grande schermo orizzontale frange la luce del sole con un mucharabi che attutisce la luce e il calore, inducendo il movimento del pubblico a temperarsi e a rendersi disponibile per una nuova vita parallela. Ed ecco il miracolo, in pochi momenti solenni della vita del paese e della capitale, la piazza improvvisamente si ferma, totalmente. Lo spazio si trasforma in uno spartito in ascolto, e le istanze di progetto esplorano il nuovo temporaneo, dal rumore di pause di silenzio e di comizio.

Gli spazi pubblici sono studi molto attenti all'evoluzione di ogni settore creativo che riguardi l'habitat, ma architettura, urbanistica, design dicono e non dicono della missione estetica del giardino e del paesaggio, quando e perché questi filtri critici aprano o chiudano a loro volta.
Noi abbiamo inseguito un paesaggio che sia nuovo e antico al tempo stesso. Nella creazione del nostro habitat quotidiano quali paesaggi potranno dirsi intangibili? È davvero un sogno impossibile che l'architettura, l'urbanistica, il design, il paesaggio, possano diventare gli spartiti di una narrazione coordinata del quotidiano? E così pure la poesia, la grafica, la scrittura, è possibile che possano avere un ruolo più importante nella società, nelle piazze, nelle strade, lungo i fiumi? Questa importanza avrebbe un valore sociale e economico, quindi politico rilevante. L'intangibilità ha in questo l'ambizione di fissare e non disperdere il frutto di tante battaglie e parimenti il pericolo di trincerarsi in una mentalità di difesa, un gioco che se prolungato oltre il lecito finisce con il macinare retorica, assumere un ruolo perdente.

\section{Roma o cara. Il valore Città}

Mi si dirà che in fondo ogni essere umano è fatto così, una mescolanza di termini simili e opposti che si bilanciano in un equilibrio ponderale. Vi è un lungo elenco di città ideali che spingono e comprimono i loro caratteri, portandoli a volte ad essere dei paradossi, e vi è un altrettanto lungo elenco di altre città, che invece filtrano e assorbono qualsiasi segno e accolgono qualche architettura di pregio solo ogni tanto, come una stravaganza. Ci sono città reazionarie e città anarchiche, ciascuno di noi ha i suoi modelli, ma bisogna cercare di concentrare gli sforzi in modo di condividere obiettivi con nuove qualità di orientamento e qualità di centralità. Se vogliamo come riferimento un landmark, un bell'albero potrà esserci di aiuto, o un'altra folie che si faccia carico del senso di questo luogo in modo imprevedibile.

Dei giardini inglesi: è uno dei testi politici più importanti dell'inizio del Ottocento, opera di Ercole Silva, un intellettuale che ha una cultura e un talento straordinari. In quell'epoca Milano era uno dei luoghi di elaborazione politica più avanzati d'Europa. Fra i documenti politici più interessanti è la descrizione del parco pubblico inglese, il cui statuto è un program- 
ma di salute pubblica, ricco di contenuti di estimo e di welfare e la ricchezza dei giardini di acclimatazione è dettata da un interesse botanico che recentemente è stato ripercorso dall'expo di Milano 2015 sui temi dell'alimentazione.

Parlando di valori intangibili nel paesaggio pensiamo a tutti quei nostri diritti e doveri che sono indispensabili per celebrarne il significato e la rappresentatività, per tutelarne la manutenzione, l'essenza fisica e morale della sua sostenibilità, assicurarne i processi di gestione, valorizzarne le vocazioni, crearne i presupposti per un armonioso sviluppo che sia in equilibrio fra tradizioni del luogo e visioni del futuro di una sua possibile trasformazione.

Ma le cose sono più semplici e al tempo stesso più complesse, perché i paesaggi, come dice Lucien Kroll, “...non sono innocenti”, sono anch'essi in perenne agone fra Lucifero e Michele, gli arcangeli dell'eterna lotta fra il male e il bene. Certo noi vogliamo il buono, il bello e il giusto, ma abbiamo subito bisogno di una guida per i nostri passi, non solo di quella di Virgilio per la discesa nell'Ade, ma di un potente sussidio filosofico e di un potente sussidio rappresentativo, e sappiamo non bastarci ancora, mentre sopravvengono altri campi del sapere, segnatamente conoscenze tecniche e economiche. Ma naturalmente tutto ciò si deve basare su una rinnovata sensibilità che orienti tutti quei "... tagli nella sostanza del mondo" (II Reno di Hölderlin, da Roberto Pasini) che evocano un paesaggio come sviluppo di una intuizione creativa.

"Libertà" è l'unico valore che anche da solo può rappresentare caso per caso quel sentimento cui concorrono individui e comunità, perché sostie- ne la loro identità affermando l'appartenenza a una società e a una cultura in seguito a una dichiarazione di volontà, un sentimento che è il più diretto, l'amore per la propria indipendenza, un patto evidentemente non contrattabile oltre ai termini di un suo statuto, che varia nel tempo, ma che può essere confermato in ogni momento con precisione esprimendolo con una volontà esplicita. II progetto più recente che vi propongo è il Barranco di Tenerife di Juan Manuel Palerm e di Leopoldo Tabares de Nava, con un capolavoro di equilibrio in temi fra loro quanto mai potenzialmente ostili. II caso fra mille è quello di un torrente che da monte a mare attraversa quartieri abitati per quasi tre chilometri, prevedendo anche il passaggio di una superstrada, il tutto partendo dal parere che bisognasse evitare in modo fermo la soluzione originaria di tombare il torrente, come si vede una sciarada apparentemente improponibile eppure invece perfettamente risolta da un progetto che sembra saper cogliere una sfida rilanciando, nessuna mitigazione, un racconto rapido e felice, la dimostrazione di uno spirito del tempo che esso stesso è rimasto un principio intangibile: viva la libertà!

Questo principio apparentemente così complesso è alla base di importanti continue variazioni dell'assetto fisico del territorio e riguarda l'evoluzione di quelle qualità di orientamento e di centralità che definiamo come caratteristiche dei paesaggi. In realtà è anche molto complicato, essendo i valori rappresentati articolati in più problematiche, su uno spettro di azioni, nei casi che io porgo alla vostra attenzione, si tratta di opere d'arte che sono state dichiarate intangibili, come lo è la dichiarazione dei diritti dell'uomo. 


\section{Usi civici}

L'idea di paesaggi intangibili, opere che fanno parte di un patrimonio condiviso che a nessun costo può essere oggetto di alcun atto riduttivo, né fisico né virtuale, sembra tornare di attualità. II motivo potrebbe essere un ritorno di impegno politico nelle vicende delle trasformazioni del territorio e in particolare una chiamata di responsabilità che recentemente ha riportato la Convenzione Europea del Paesaggio a proporsi come garante dei complessi processi attuativi che vanno dall'ideazione di un'opera alla sua assimilazione da parte di una comunità attraverso un confronto partecipativo.

Dopo le Istituzioni di Diritto romano, nell'alto medio evo gli usi civici si presentano come una serie di disposizioni di opportunità, di necessità e di mutuo soccorso fra piccole comunità e poteri ecclesiastici e nobiliari, riconoscimento di servitù di passaggio, uso di dispositivi come un pozzo, o un canale, o un mulino, partecipazione alla quota di un raccolto... Bravi notai trovano ancora oggi tracce di usi civici che sono attivi, dimenticati nelle pieghe di territori che appaiono come terre di leoni. È successo a me quando alla fine del secolo scorso mi occupai del Parco di Monte Mario, una sorta di archeologia che scorre nelle vene di una terra antica, una impressione che mi ha commosso, ricordandomi Victor Hugo quando diceva che se "I'avvenire è una porta, il passato ne è la chiave".

Non è commovente? Scavando, accanto a pezzi di statue, anfore e vasi, e fossili vegetali affiorano in superficie anche documenti di natura imprevedibile.

\section{Inoltre, e non in subordine}

Diamoci dunque la capacità di scrivere e leggere, e sempre di più di saper anche ascoltare le voci dei luoghi nei quali si intende intervenire. Spesso nel mio lavoro cerco di definire i caratteri di un paesaggio intangibile che sia nuovo e antico al tempo stesso, colto nel momento che si dispieghi nello spazio. Cerco, ad esempio, come Kundera, in una stessa dimensione narrativa, di passare da un peso corale beethoveniano alla leggerezza e alla fragranza di un haiku, o viceversa. Ma fino a che punto può continuare a essere nitida la nostra scrittura prima di diventare un campo sempre più serrato e ordinato, poi denso e saturo, e infine neutro? II pericolo è di generare dislessia e daltonismo, esattamente come quel campo di disordine da cui è partita questa stessa nostra esigenza. Ma questo, forse, è nient'altro che un pensiero banale. 